\title{
MANET: A SURVEY ON MACHINE LEARNING- BASED INTRUSION DETECTION APPROACHES
}

\author{
Safaa Laqtib ${ }^{1}$, Khalid El Yassini ${ }^{1}$ and Moulay Lahcen Hasnaoui ${ }^{2}$ \\ ${ }^{1}$ Informatics and Applications Laboratory (IA), Department of Mathematics and \\ Computer Science, Faculty of Sciences, Moulay Ismail University, Meknes, \\ Morocco \\ ${ }^{2}$ Research Team: ISIC ESTM, L2MI Laboratory, ENSAM, Moulay Ismail \\ University, Meknes, Morocco \\ laq.safaa@gmail.com, khalid.elyassini@gmail.com, myhasnaoui@gmail.com
}

\begin{abstract}
A Mobile Ad-hoc Network (MANET) is infrastructure less network which is a collection of moving nodes connected dynamically in an arbitrary manner. Machine learning (ML) is the scientific study of algorithms and statistical models that computer systems use to effectively perform a specific task without using explicit instructions, relying on patterns and inference instead. It is seen as a subset of artificial intelligence. MANETs are more susceptible to the security attacks because of the node mobility which imposes a set of challenges security issues. To tackle these security issues, such as the use of encryption and authentication techniques, have been proposed as a first line of defense to reduce the risk of security problems. However, such risks cannot be completely eliminated, there is a strong need of intrusion detection systems (IDS) as a second line of defense for securing MANET. An intrusion-detection system (IDS) can be defined as the tools, methods, and resources to help identify, assess, and report unauthorized or unapproved network activity. Machine learning based intrusion detection approaches must be deployed and elaborated to facilitate the identification of attacks and enables system to make decisions on intrusion while continuing to learn about their mobile environment. In this paper, we present the most well-known models for building intrusion detection systems by incorporating machine learning in the MANET scenario.
\end{abstract}

Keywords - MANET, Attack, Machine learning, intrusion detection system IDS

\section{INTRODUCTION}

Mobile Ad-Hoc Network (MANET) comprise of a group of autonomous nodes that form a dynamic, multi-hop radio network in decentralized and cooperative way. Due to its unique characteristics, this technology has been used to support communications in situation where it may be impossible to deploy infrastructure networks, such as military battlefields, disaster recovery sites and commercial sectors. due to nodes mobility and dynamic topology that is frequently change, MANET are very susceptible to a variety of attacks such as eavesdropping, routing, packet modification, etc. [1].

MANETs are substantially preferable to different complex distributed threats, which mostly address the network and data link layer of the protocol stack. because each packet must be passed through intermediate nodes quickly, which packet must travel to the destination from the source. Malicious routing attacks may target the routing discovery or

Received: April 7, 2019

Reviewed: May 13, 2019

Accepted: June 3, 2019

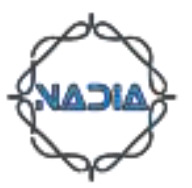


maintenance phase by failing to follow the routing protocol specifications. recent papers have identified more sophisticated and subtle routing attacks, such as the blackhole (or sinkhole) [2], Byzantine [3], and wormhole attacks. Currently routing security is one of the hottest research areas in MANET [4], Using an Intrusion Detection System (IDS) is an effective way to identify when an attack occurs in a MANET.

For the above reasons, it is very important to deploy in MANET as a second line of defense an intrusion detection system. Intrusion detection systems (IDS) are a mechanism for monitoring and investigating events occurring in a computer system. An IDS incorporates methods for modeling and discovering abnormal behaviors and complex techniques. They try to determine whether or not the network is going through any malicious activity. This is typically accomplished by gathering data automatically from a variety of systems and network sources and then analyzing the information for potential security issues [5].

Traditional techniques of intrusion detection and prevention, such as firewalls, access control mechanisms, and encryption, have several limitations in fully protecting networks and systems from increasingly sophisticated attacks such as service denial. In addition, the majority of systems built on such techniques suffer from high false positive and false negative detection rates and lack of continuous adaptation to changing malicious behaviors. Therefore, Machine learning helps to easily perform data summary and visualization, the goal is to make it easier for security professionals to identify system weaknesses and flaws. In order to improve detection rates and adaptability, several Machine Learning (ML) techniques have been applied to the problem of intrusion detection. These techniques are often used to maintain up - to - date and comprehensive knowledge bases of the attack [6].

The remainder of this paper is organized as follows. First, Section 2 is dedicated to discuss Security attacks in MANET. Then, Section 3 describes the Intrusion detection system (IDS) Types. Section 4 provides a brief survey of Intrusion detection system (IDS) In MANET. The notion of Machine learning Types then presented in Section 5. The Machine learning based IDS: MANET suitability is discussing in Section 6. Finally, concluding remarks and is presented in Section 7.

\section{SECURITY ATTACKS IN MANET}

MANET attacks can be approximately classified into two main categories, namely passive attacks and active attacks, depending on the means of attack. [7] [8]. A passive attack gets data exchanged in the network without interrupting the communication operation, whereas an active attack involves interruption of information, Modification or manufacturing, thus disrupting the MANET's normal functionality. Table 1 illustrates the general taxonomy of MANET security attacks. The eavesdropping, traffic analysis, and traffic monitoring are examples of passive attacks. The attacks can also be classified into two categories according to the domain of the attacks, namely external attacks, and internal attacks. External attacks are performed by nodes that are not part of the network's domain. Internal attacks are from compromised nodes that are part of the network in fact. Internal attacks are more severe than external attacks [9].

Recent research papers have identified more sophisticated and subtle routing attacks. The typical examples described many attacks.

\subsection{WORMHOLE ATTACK}

At one location in the network, an attacker records packets and tunnels them to another location. When routing control messages are tuned, routing can be severely disrupted. This tunnel is referred to as a wormhole between two colluding attackers. [10]. 
Wormhole attack can be done with single node also but generally two or more malicious node connects via a wormhole-link. In fig 1, Node X and $\mathrm{Y}$ performing wormhole attack.

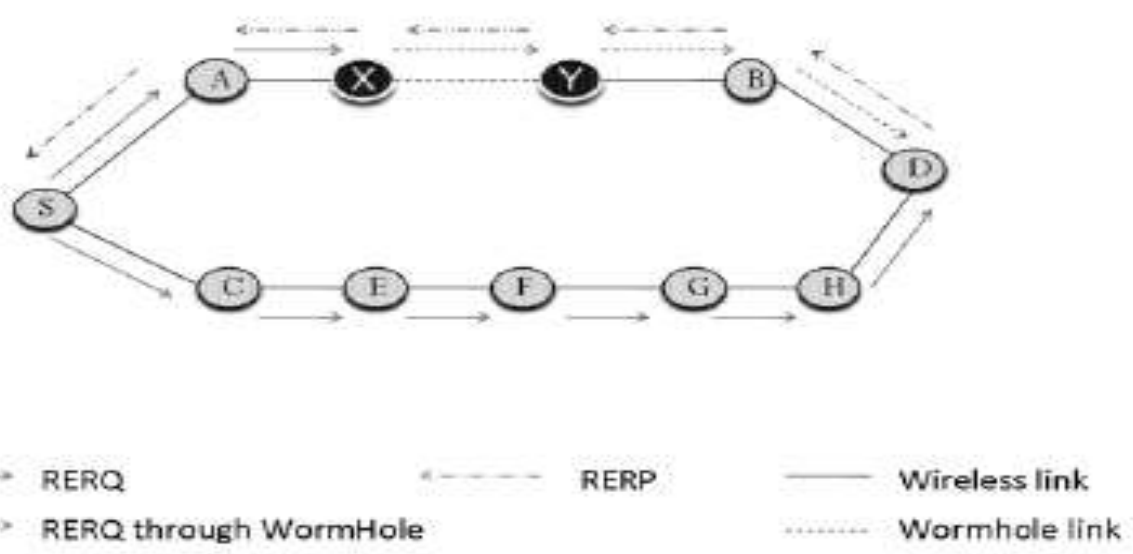

Fig. 1 Worm-Hole Attack

\subsection{BLACKHOLE ATTACK}

There are two properties to the blackhole attack. First, the node uses the mobile ad hoc routing protocol, such as AODV, to advertise itself as having a valid route to a destination node, despite the spurious route, with the intention of intercepting packets Second, the attacker consumes the intercepted packets without any forwarding. However, the attacker runs the risk that neighboring nodes will monitor and expose the ongoing attacks. There is a subtler form of these attacks when an attacker selectively forwards packets. An attacker deletes or modifies packets originating from certain nodes while leaving the data from the other nodes untouched, limiting the suspicion of their wrong doing, fig 2 presents an example of Black-Hole Attack [11].

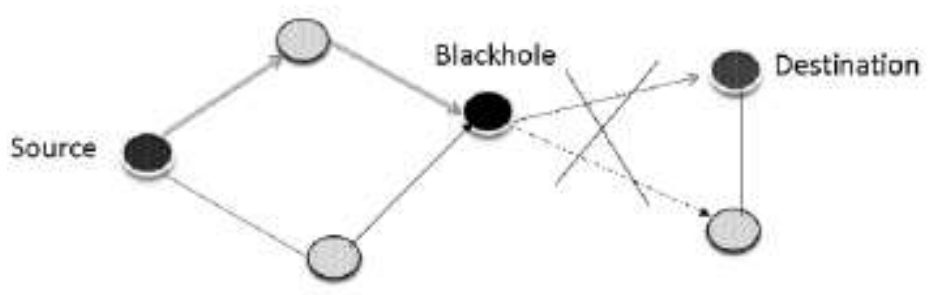

Fig. 2 Black-Hole Attack

\subsection{RUSHING ATTACK}

A wormhole is formed by two colluded attackers using the tunneling procedure. If there is a fast transmission path between the two ends of the wormhole (e.g. a dedicated channel shared by attackers), the tunneled packets can propagate faster than the ones via a normal multi-hop route. This is the rushing assault. The rushing attack can act as an effective denial - of - service attack against all MANET routing protocols currently proposed on demand, including protocols designed to be safe, such as ARAN and Ariadne, fig 2 presents an example of Rushing Attack [12][13]. 


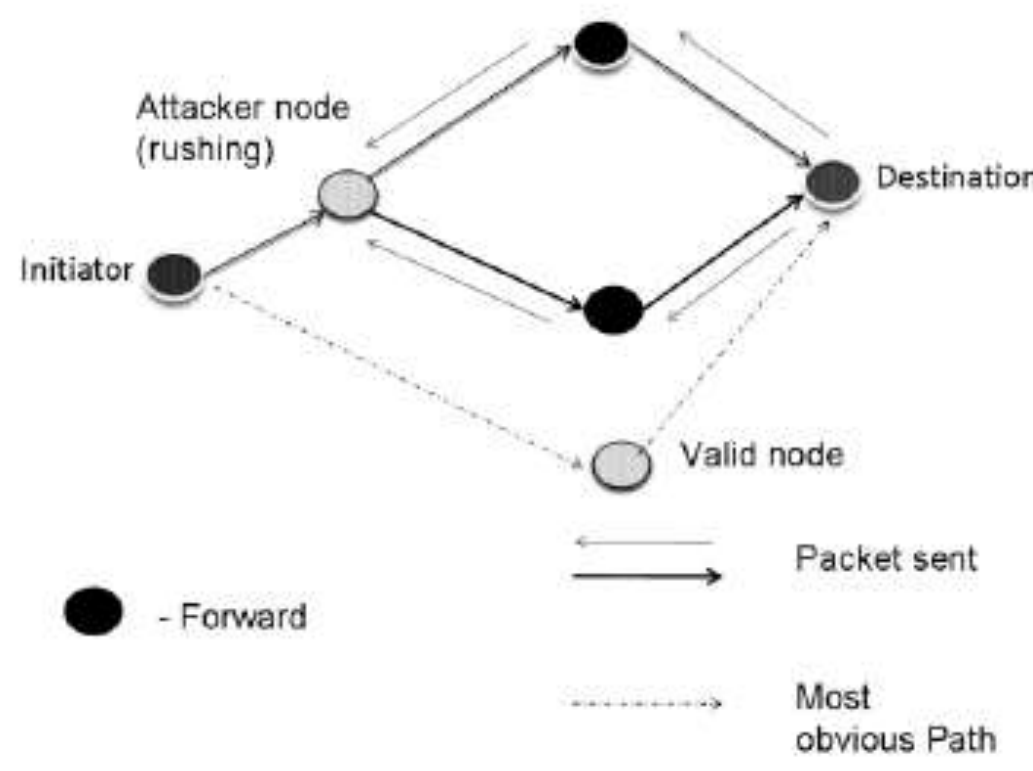

Fig. 3 Rushing Attack

\subsection{BYZANTINE ATTACK}

A compromised intermediate node works alone or a set of compromised intermediate nodes works in collusion and performs attacks such as creating routing loops, forwarding packets through non - optimal paths or selectively dropping packets resulting in routing services being disrupted or degraded [13][14].

\subsection{SYN FLOODING ATTACK}

The SYN flooding is A denial - of - service attack. The attacker creates a large number of half-opened TCP connections with a victim node, but never completes the handshake to open the connection completely. For two nodes to communicate using TCP, a threeway handshake must first be used to establish a TCP connection. The three messages exchanged during the handshake, shown in Fig 4, enable both nodes to learn that the other is ready to communicate and agree on initial sequence numbers for the conversation. [15].

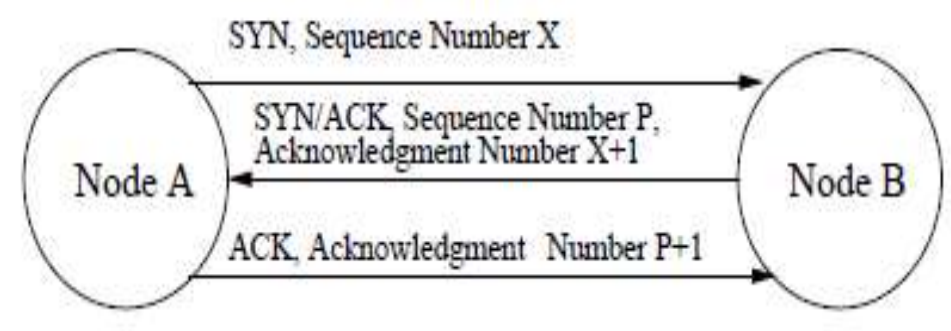

Fig. 4 TCP Three-way Handshake

A malicious node sends a large quantity of SYN packets to a victim node during the attack, spoofing the SYN packets ' return addresses. The SYNACK packets are sent from the victim immediately after the attacker receives the SYN packets and the victim is waiting for the ACK packet's response. The half-open data structure remains in the victim 
node without any response from ACK packets. If the victim node stores these half-opened connections in a fixed - size table while waiting for the three-way handshake to be recognized, all of these pending connections could overflow the buffer, and no other legitimate attempts to open a connection could be accepted by the victim node. There is usually a time-out associated with an outstanding connection so that the half-open connections eventually expire and the victim node recovers. However, malicious nodes can simply continue to send packets requesting new connections faster than pending connections expire. [16].

\subsection{DENIAL OF SERVICE}

Denial of service (DoS) attacks could be launched from several layers. In the physical layer, an attacker can use signal jamming that disrupts normal communication. Malicious nodes can occupy channels at the link layer through the capture effect, taking advantage of the binary exponential scheme in MAC protocols and preventing channel access to other nodes. At the network layer, the routing process may be interrupted by modification of the routing control packet, selective drop, table overflow, or poisoning. SYN flooding, session hijacking and malicious programs can cause DoS attacks on transport and application layers [17][18].

\subsection{DENIAL OF SERVICE (PING OF DEATH)}

Ping of Death Attack is also a Denial of Service Attack that uses unformatted or improperly formatted ping. If the ping bytes are greater than 65535, this attack may occur. Linux, Windows OS are vulnerable for this attack [19][20].

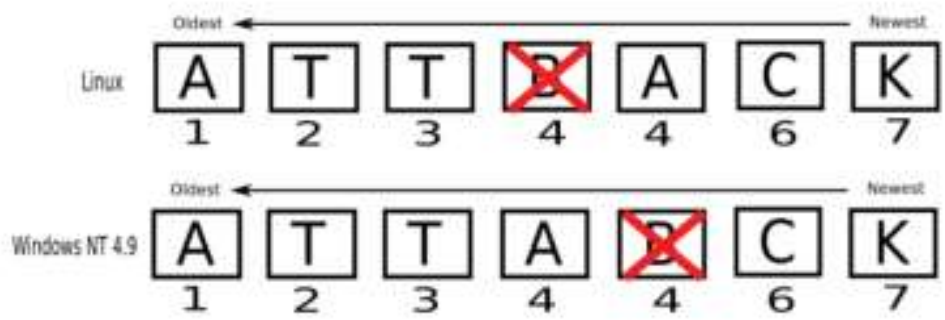

Fig. 5 Overlapping Packet

Fig. 5, in number 4 slot there are two packets arrived at a time and one packet is denied. Here it shows Linux and Windows OS systems structure.

\section{INTRUSION DETECTION SYSTEM (IDS) TYPES}

IDS can be classified into two types- depending on data collection mechanism and detection techniques. Types of IDS depending on the data collection mechanism includes Network based IDS (NIDS) and Host based IDS (HIDS). Network-based IDS runs on a network gateway or router and captures all the network traffic that passes through it. Detecting an attack from outside will be useful. As there is no central coordination, this is not suitable for MANET. A host-based IDS captures a particular host's local network traffic. It's better to detect an inside attack. Although, on the other hand, there are mainly two types of IDS in the detection techniques category [21], are as follows:

\subsection{ANOMALY-BASED DETECTION SYSTEMS}

The normal behavior or daily activities of a user are kept within the system in this type of detection system. Whenever the user or the attacker does any activity, the system 
compares this activity with the data stored and then handles the evaluation - based activity, whether or not it is an intrusive activity, and responds to the system.

\subsection{SIGNATURE BASED DETECTION SYSTEMS}

Signature-based IDS refers to the detection of attacks by looking for specific patterns, such as byte sequences in network traffic, or known malicious instruction sequences used by malware. This terminology originates from anti-virus software, which refers to these detected patterns as signatures. Although signature-based IDS can easily detect known attacks, it is difficult to detect new attacks, for which no pattern is available There is another perspective of reaction-based classification of the IDS system. There are two types of IDS system one is active and another is passive, Active IDS (see Fig 6)can take immediate action and notify the administrator of any attack. Passive IDS, on the other hand, stores the log intrusion details and then notifies the manager.

Based on usage of frequency can classify an IDS system Which are offline IDS and Online IDS. Offline IDS is used to analyze pre-logged data to detect any attack where Online IDS is offline IDS analyzes pre-logged data to detect intrusion, but Online IDS uses new data to detect an attack.

But in general, there are only two types of IDS: one is Signature based and the other is Anomaly based. [22].

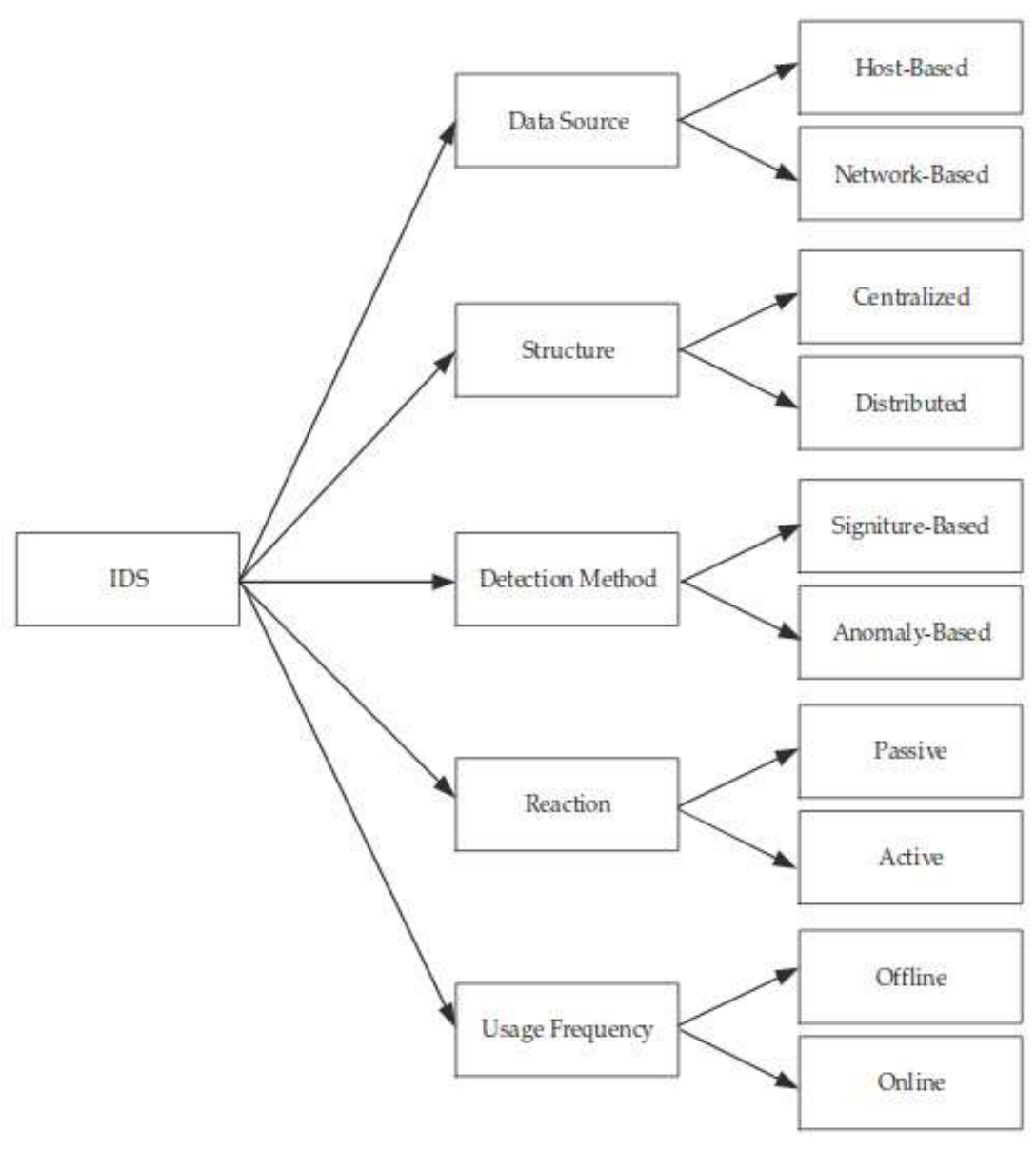

Fig. 6 Basic Design of IDS System 


\section{INTRUSION DETECTION SYSTEM (IDS) IN MANET}

The objective of an intrusion detection system (IDS) is to detect attacks or malicious activities in a standalone or network system by continuously monitoring the traffic audit trail. Mobile ad hoc networks (MANETs) are composed of mobile wireless nodes that are dynamically self - organized in a given network, so nowadays IDS has become an integral part of mobile nodes in order to provide the network with deep defense security. Despite the high degree of flexibility and the wide range of applications offered by MANETs, the vulnerability inherent in them increases security concerns. To safely utilize Manets ' cooperative and dynamic nature, highly efficient and effective security mechanisms are needed. However, Prevention of intrusion cannot be used to eliminate any possible intrusion. It can only be used as the defense's first layer. Detection of intrusion using classification algorithms can make it easier to discriminate "normal" from "abnormal" behavior and thus detect intrusions [23]. The same can therefore be used as a second layer of defense and can therefore serve as a major block in the MANET communication system. Many intrusion detection systems have been proposed for wired network Where all traffic passes through switches, routers or gateways to facilitate the implementation of IDS on these devices. While MANET, on the other hand, does not have all of these devices and because of its open medium, any user can access it. The current wired network IDS technique cannot, therefore, be implemented directly on MANET. Mainly three types of IDS techniques [24] can be used on MANET.

\subsection{STAND ALONE INTRUSION DETECTION SYSTEM}

In this system, to determine intrusion, the intrusion detection system runs independently on the individual node. All decisions made about a particular activity depend solely on information collected at its own node, as there is no collaboration between nodes in the network. Therefore, there is no transfer of information. Even, as no alert information is transferred, a node in the same network does not have any information about the other nodes in the network. Because of its limitations, this model is not efficient, it can be used effectively in a network where all nodes already have an IDS installed. Compared to multi - layered network infrastructure, this system is also suitable for single layer network. Since the information available on any single node is not sufficient to detect intrusions, this system has not been chosen as MANET IDS [25][26].

\subsection{DISTRIBUTED AND COOPERATIVE INTRUSION DETECTION SYSTEM}

In this architecture, each node has an IDS agent that locally detects intrusions and collaborates with neighboring nodes for global detection whenever there is indeterminate evidence and a broader search is required. When the intrusion is captured, either a local response (e.g. alerting the local user) or a global response may be issued by an IDS agent. Each node is involved in the method and response of intrusion detection as having an IDS agent running on it [27]. An IDS agent is responsible for detecting and collecting local information and data in order to identify any attack if an attack occurs in the network, as well as taking an independent response [28]. However, when the evidence is non conclusive, neighboring IDS agents also cooperate in global intrusion detection. This system, like stand - alone IDS, is also more suitable for flat network systems, not multi layer systems [29].

\subsection{HIERARCHICAL INTRUSION DETECTION SYSTEM}

Hierarchical IDS system Expand the distributed and cooperative IDS system functions and have been implemented for multi - layer network infrastructures where the network is divided into various small networks known as clusters [30]. Usually, each cluster head has more functionality than other cluster members, such as transmitting data packets to 
other clusters. We can therefore say that these cluster heads work in some way as central point's similar to wired network control devices such as routers, switches or gateways. The multi - layering concept applies to intrusion detection systems where there is a proposal for hierarchical IDS. Each IDS agent runs on a specific member node and is responsible for its node, i.e. monitoring and deciding on intrusions detected locally. A cluster head is responsible for their node locally as well as globally for their cluster, such as monitoring network traffic and announcing a global response when detecting network intrusion [31].

\section{MACHINE LEARNING TYPES}

Machine learning (ML) is part of the altricial intelligence. Machine learning is used to make computers seem smart, and definition machine learning is like teaching computers to learn, so instead of programming every single outcome we do, we take a computer and feed it a bunch of data, and it will learn from that data and improve over time. Machine learning is becoming so popular now because we have so much data in our hands. So, we're turning to computers to do the analysis for us, and in something called data analytics this is very popular. Our data well we can add some discipline by having predictive data analytics. Machine learning algorithms (see Fig 7) fall into three categories: supervised learning, unsupervised learning and reinforcement learning [32].

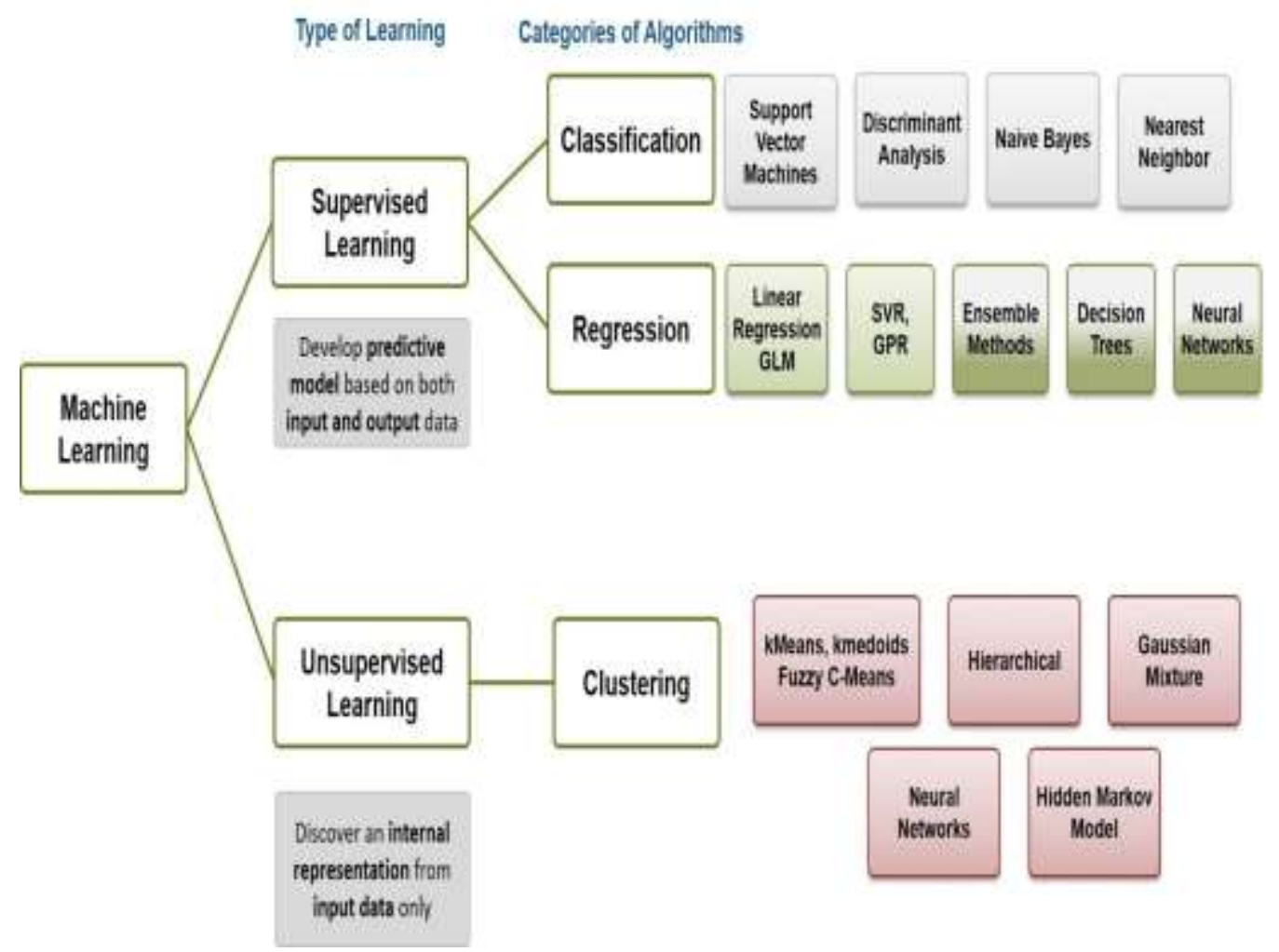

Fig. 7 Taxonomy of Supervised and Unsupervised Techniques

\subsection{SUPERVISED LEARNING}

Training is supervised as a name that indicates that training can be a teacher or instructor in the presence of a supervisor. In this case, the data in which the data label is indicated must be specified or trained, that is, the device in which certain data have already been marked with the correct answer. Learning monitoring is a general concept that applies to scenarios in the real world. The controlled learning algorithm analyzes the 
learning data and generates a function classifier (if discrete output) or a function of regression (if continuous output). There's a lot of supervised learning algorithms. Artificial Neural Network (ANN), Support Vector Machine (SVM), K-Nearest-Neighbors (KNN), Ensembles classifiers (Bagging), Decision Trees (C4.5, ID3, CART, Random Forrest), Linear Classifiers (Naive Bayes (NB)) classifiers are popular supervised learning algorithms [33]. Table I below illustrated the taxonomy of supervised ML methods, in terms of their pros and cons.

Table I. Taxonomy of Supervised Machine Learning Based Intrusion Detection

\begin{tabular}{|c|c|c|}
\hline Technique & Characteristic/ Advantages & Limitations/ Challenges \\
\hline $\begin{array}{l}\text { Artificial neural } \\
\text { Network }\end{array}$ & $\begin{array}{ll}- & \text { Difficulties in updating new } \\
\text { - } & \text { information, } \\
- & \text { Bnable to detect novel intrusion } \\
- & \text { Comprises of three phases: data } \\
- & \text { collection, model training, testing to } \\
- & \text { identify the malicious user. } \\
- & \text { able to work in limited, noisy and } \\
- & \text { incomplete dataset. } \\
- & \text { Easy Leaning that does not need to be } \\
& \text { re programmed. }\end{array}$ & $\begin{array}{ll}\text { - } & \text { High processing time. } \\
- & \text {-Slow training process so may } \\
& \text { not suitable highly mobile } \\
- & \text { networks. } \\
- & \text { - Data overfitting happens } \\
& \text { during training }\end{array}$ \\
\hline Decision Tree & $\begin{array}{ll}- & \text { Suitable for large datasets. } \\
- & \text { Detection accuracy is high } \\
\end{array}$ & $\begin{array}{ll}\text { - } & \text { constructing decision tree } \\
\text { involves high computation. }\end{array}$ \\
\hline $\begin{array}{l}\text { Bayesian } \\
\text { Network }\end{array}$ & $\begin{array}{ll}\text { - } & \text { Based on graphical model that } \\
\text { generates } \\
\text { - } \\
\text { probabilistic relationships among } \\
\text { interested variables. } \\
\text { - } \quad \text { Prior knowledge can be incorporated. } \\
\text { - } \quad \text { Fast, scalable and use nonparametric } \\
\text { means of extracting predictive } \\
\text { information. } \\
\text { - } \quad \text { It can handle incomplete datasets. } \\
\text { Efficient for avoiding over fitting of } \\
\text { data. }\end{array}$ & $\begin{array}{ll}\text { - } & \text { Difficult to handle continuous } \\
\text { features. } \\
\text { - } & \text { It is computationally expensive } \\
\text { process } \\
\text { - } \\
\text { as it requires extra power } \\
\text { which tends to } \\
\text { - } \quad \text { be slow for large features sets. } \\
\text { - } \\
\text { May not work well if prior } \\
\text { knowledge is not correct. }\end{array}$ \\
\hline $\begin{array}{l}\text { Support vector } \\
\text { Machine }\end{array}$ & $\begin{array}{ll}\text { - } & \text { Place input vector into a greater } \\
\text { dimensional feature space for } \\
\text { obtaining } \\
\text { - } \\
\text { - } \quad \text { outliers. Can solve non-linear } \\
\text { classification and } \\
\text { - } \quad \text { regression. } \\
\text { - } & \text { Automatically avoids data overfitting. }\end{array}$ & $\begin{array}{ll}\text { - } & \text { Number of misclassified } \\
\text { samples and } \\
\text { the width of a decision } \\
\text { boundary gives the trade-off. } \\
\text { - } \quad \text { Training takes long time. }\end{array}$ \\
\hline
\end{tabular}

\subsection{UNSUPERVISED LEARNING}

Uncontrolled training is the study of tasks performed by supervisors without supervision. Tasks are performed on the basis of past experience with unsupervised learning. Training involves data learning, but not goals and predictions. Unsupervised learning refers to the problem of unmarked data finding a secret structure. There is no clear objective or environmental assessment for each entry, unlike supervised learning. Rather, an impartial apprentice gives rise to previous prejudices that should include aspects of the input composition in the output. There are many learning algorithms that are not supervised. Clustering and algorithm of deduction [34]. 
The detailed of taxonomy of machine learning based anomaly intrusion detection techniques have been illustrated below for unsupervised ML methods in the Table II in terms of their pros and cons.

\subsection{REINFORCEMENT LEARNING}

Reinforcement Learning (RL) is a type of machine learning technique that enables an agent to learn in an interactive environment by trial and error using feedback from its own actions and experiences, there are many reinforcement learning algorithms. Q-Learning, Temporary Difference (TD), Deep Adversarial Networks and Algorithm for Markov Decision [35].

Though both supervised and reinforcement learning use mapping between input and output, unlike supervised learning where feedback provided to the agent is correct set of actions for performing a task, reinforcement learning uses rewards and punishment as signals for positive and negative behavior. As compared to unsupervised learning, reinforcement learning is different in terms of goals. While the goal in unsupervised learning is to find similarities and differences between data points, in reinforcement learning the goal is to find a suitable action model that would maximize the total cumulative reward of the agent. The figure below represents the basic idea and elements involved in a reinforcement learning model.

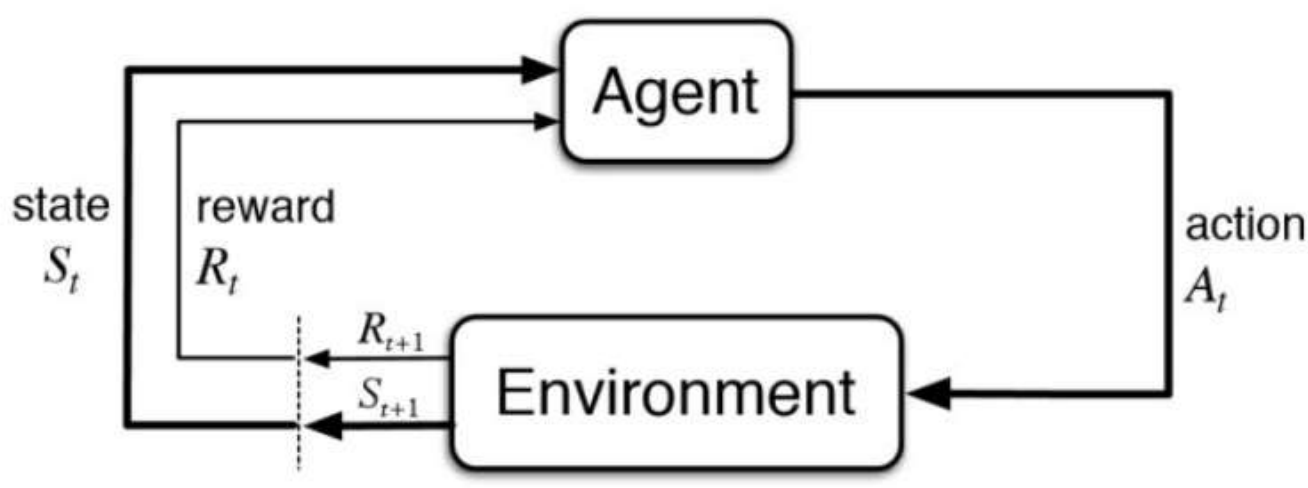

Fig. 8 The agent-environment interaction in a Markov decision process.

Some key terms that describe the elements of a RL problem are:

Environment: Physical world in which the agent operates

State: Current situation of the agent

Reward: Feedback from the environment

Policy: Method to map agent's state to actions

Value: Future reward that an agent would receive by taking an action in a particular state

Q-learning is a commonly used model free approach which can be used for building a self-playing PacMan agent. It revolves around the notion of updating $\mathrm{Q}$ values which denotes value of doing action $a$ in state $s$. The value update rule is the core of the Qlearning algorithm.

\subsubsection{DEEP Q NETWORK (DQN)}

Deep Q-Networks which use Neural Networks to estimate Q-values. But DQNs can only handle discrete, low-dimensional action spaces. DQN leverages a Neural Network to estimate the Q-value function. The input for the network is the current, while the output is the corresponding Q-value for each of the action. In 2013, DeepMind applied DQN to Atari game, as illustrated in the below Fig 9. The input is the raw image of the current 
game situation. It went through several layers including convolutional layer as well as fully connected layer. The output is the Q-value for each of the actions that the agent can take [36].

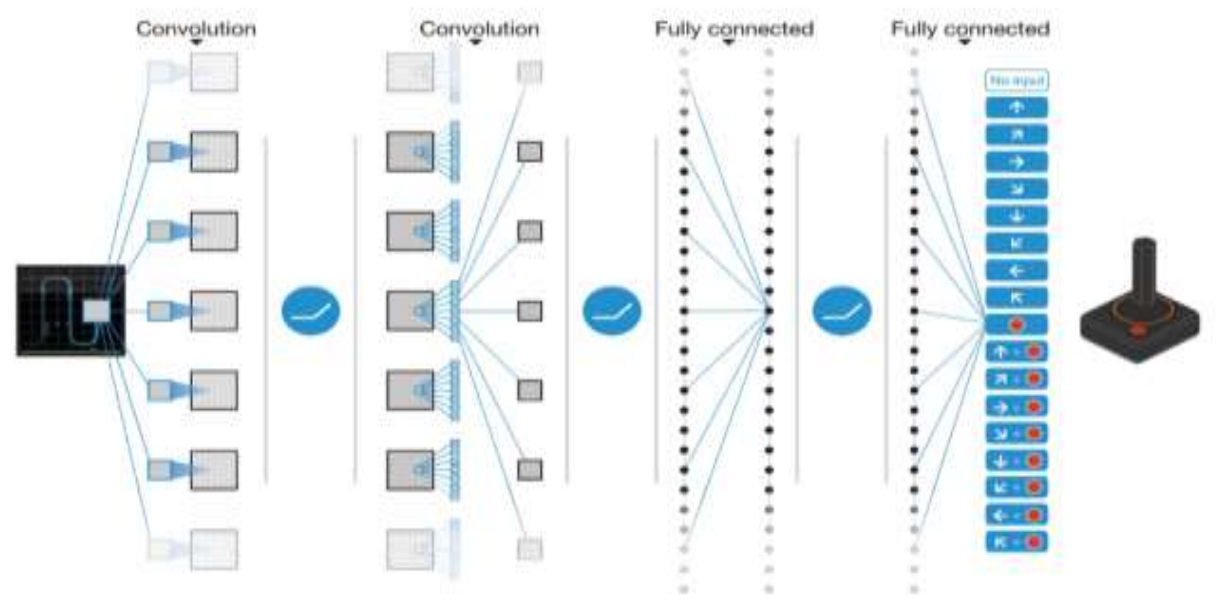

Fig. 9 Atari Example

\subsubsection{DEEP DETERMINISTIC POLICY GRADIENT (DDPG)}

DDPG relies on the actor-critic architecture (see Fig 10) with two eponymous elements, actor and critic. An actor is used to tune the parameter $\boldsymbol{\theta}$ for the policy function, i.e. decide the best action for a specific state [37].

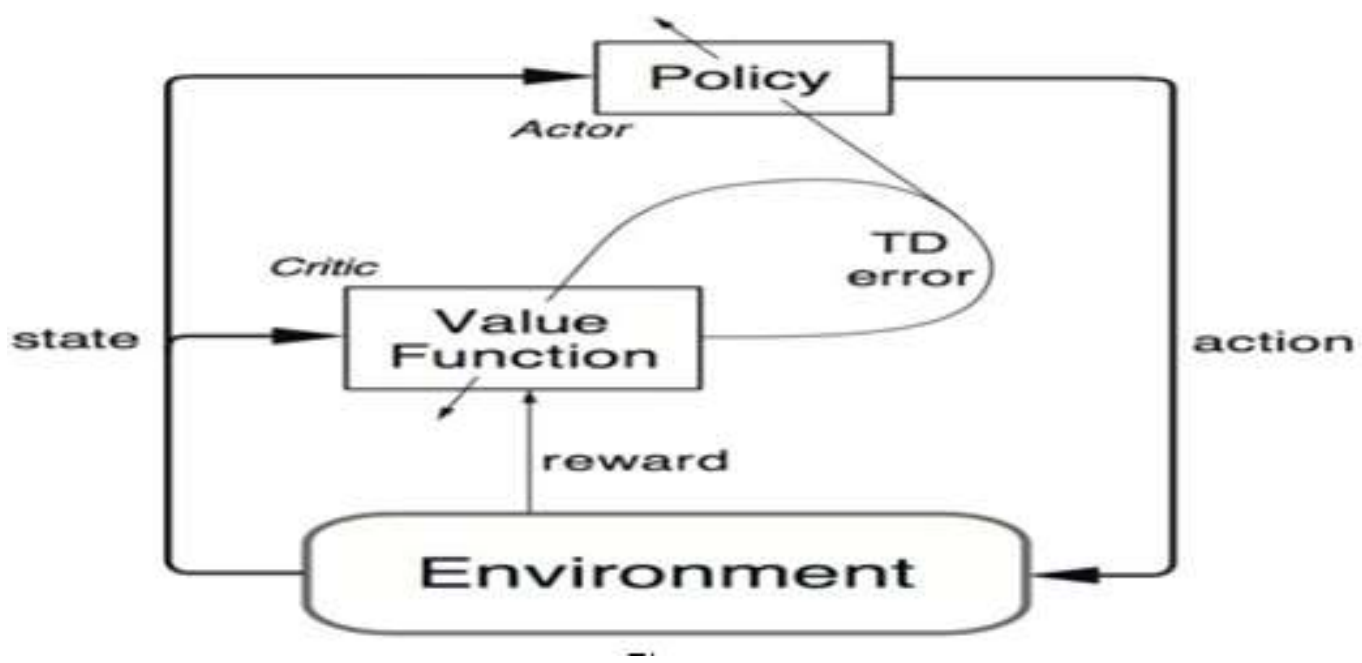

Fig. 20 Actor-Critic Architecture for Reinforcement Learning 
Table III. Taxonomy of unsupervised Machine Learning Based Intrusion Detection

\begin{tabular}{|c|c|c|}
\hline Technique & Characteristic/ Advantages & Limitations/ Challenges \\
\hline $\begin{array}{l}\text { K-means } \\
\text { Clustering }\end{array}$ & 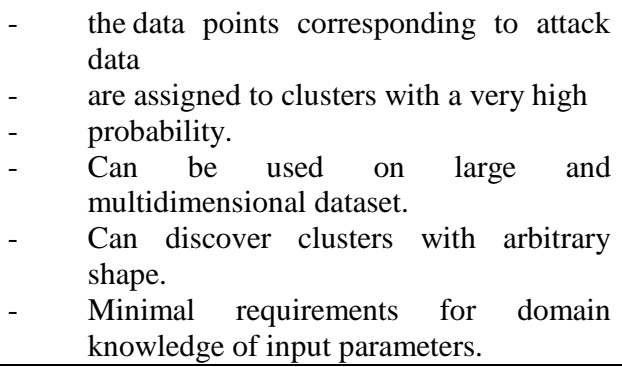 & $\begin{array}{l}\text { - Presence of outliers, may lead to } \\
\text { misclassification. Fixing number } \\
\text { of clusters at initial stages and } \\
\text { the results depend on the value } \\
\text { of mean. }\end{array}$ \\
\hline $\begin{array}{l}\text { Association } \\
\text { Rule learning }\end{array}$ & 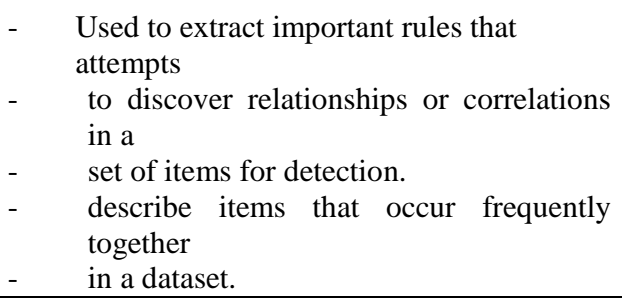 & $\begin{array}{l}\text { - it does not return } \\
\text { the same result with each } \\
\text { evaluation, because it depends } \\
\text { on the initial assignments. } \\
\text { - does not have time efficiency. }\end{array}$ \\
\hline $\begin{array}{l}\text { Hidden } \\
\text { Markov Model }\end{array}$ & 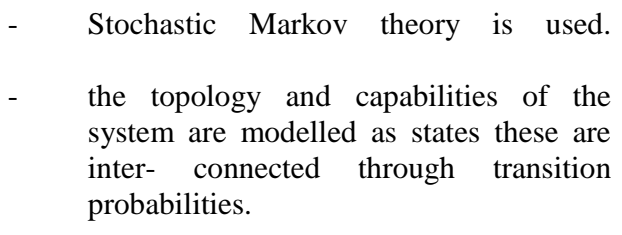 & $\begin{array}{l}\text { - Computationally } \\
\text { intensive } \\
\text { requires mechanisms to reduce } \\
\text { the algorithmic overhead. }\end{array}$ \\
\hline $\begin{array}{l}\text { Genetic } \\
\text { Algorithm }\end{array}$ & 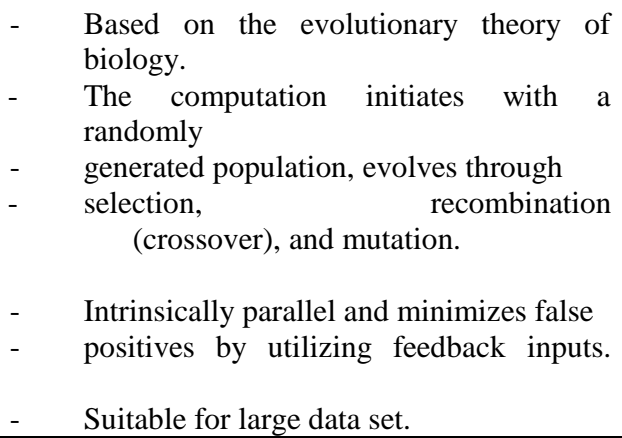 & $\begin{array}{ll}\text { - } & \text { Low Crossover rate. } \\
\text { - } & \text { High Mutation rate. } \\
\text { - } & \text { Fitness function accurate. } \\
\text { - } & \text { Cannot assure response } \\
& \text { time optimization. } \\
\text { - } & \text { Overfitting happens. }\end{array}$ \\
\hline Fuzzy Logic & $\begin{array}{l}\text { - Based on approximation and uncertainty } \\
\text { - can handle discrete and continuous } \\
\text { attributes both. } \\
\text { - Accurate and can avoid sharp boundary } \\
\text { problem }\end{array}$ & $\begin{array}{l}\text { - } \quad \text { Reduced, relevant rule subset } \\
\text { - } \quad \begin{array}{l}\text { Identification and rule } \\
\text { updating dynamically during }\end{array} \\
\text { runtime is a demanding job. } \\
\text { - } \\
\text { High resource consumption. }\end{array}$ \\
\hline
\end{tabular}

\section{MACHINE LEARNING BASED IDS: MANET SUITABILITY}

The objective of this section is to provide a better perception in MANETs for the establishment of ML - based IDS. It emerges from the comprehensive literature survey that the following points should mainly be considered when designing the IDS for the application of MANET and covered in sub - sections below:

- Appropriate selection of captured data features

- Appropriate intrusion calculation architecture 
- Development of an appropriate automated model for detection of anomalies

\subsection{FEATURE SELECTION AND EXTRACTION}

Feature selection and extraction Is the method of selecting important parameters / features from a large data set. It is essential to select significant features that should cover information from multiple types / sources in order to increase the detection rate. Before any detection model is established, the probabilistic behavior analysis of the given network is essential, which includes node mobility in a given network, the number of malicious nodes, the data sampling interval and the type of attacks. Although different protocols interpret it in a slightly different way, even under the same measure. Probabilistic network behavior analysis, some sets of features can be identified as redundant or contribute little to the process of intrusion detection, and may be omitted. Missing significant intrusion-related characteristics would make it difficult to discriminate against attacks from normal flow of traffic, However, having non - intrusion - related features may cause errors in the models and affect the functioning of the IDS. Furthermore, including a specific feature set as an input to the detection system would prevent a particular type of attack ONLY. A large number of detection system attributes, however, would result in increased processing of computation, which would have operational limitations on MANETs. The following are the wide categories of features that have been used by re-searchers for better attack detection accuracy in MANETs, are under [38]:

- $\quad$ Packet type (Data Size, ROUTE REQUEST, ROUTE REPLY, ROUTE ERROR and HELLO message)

- $\quad$ Traffic pattern

- $\quad$ Routing change

\subsection{ARCHITECTURE FOR INTRUSION COMPUTATION LOCATION}

Architecture defines a network's role of specific nodes. The intrusion detection techniques work is fundamentally independent of the architecture of the network. However, selection of suitable network architecture for IDS deployment in MANET becomes important due to its restriction on power consumption for processing. IDSs are divided into four groups according to their data collection location: standalone, distributed \& collaborative hierarchical and mobile agent. With respect to MANET application, nearly all of the IDS are deployed on distributed \& cooperative architecture and in general they also use the concept of the mobile agent. Each node contributes to the intrusion detection process through working as an IDS agent in the Distributed IDS architecture. This local IDS agent collects data from events to detect and recognize intrusions from local networks. Consumption of resources and parallel actions of nodes during the intrusion detection process may be preferred. When a likely intrusion is observed, a collaborative detection is initiated by sending alerts to other nodes and the process increases the IDS effectiveness.

\subsection{BASED DETECTION MODEL}

ML based detection model primarily learns and classify the pattern of observed behavior of the nodes using detection algorithm by taking the appropriate features as an input. Another imperative task is, therefore, the efficient detection of model building and its deployment according to the application's requirements / challenges. MANET application module design requires the best possible energy efficiency. Hence, IDS 
mechanisms should consume as little energy as possible while achieving the targeted performance. Table 5 below summarizes the comparison on the most widely used ML methods, almost none of the afore-mentioned papers that used classification algorithms contained comparison between the models, apart from. Thus, there is a lack of evidence to favor one algorithm over the others, when intrusion detection in MANETs is discussed. Moreover, no data exists on the performance of such algorithm under different traffic conditions (i.e. mobility, number of malicious nodes), and how such meta-algorithmic parameters such as the sampling interval should be designated.

Despite ML's wide - ranging successful performance in MANETs, another essential aspect of its use for intrusion detection is the correct implementation. IDS is also vulnerable by attackers aware of their use to antagonistic countermeasures. Attackers can be responsive to the details of the ML detector, e.g., choice of classifier and parameters used, and their behavior can be changed to avoid detection. Second, by adulterating the training set, reducing or eliminating its effectiveness, more prevalent attackers can actively fiddle with the ML models. An accurate evaluation of the robustness of ML techniques requires evaluation under realistic threat models [39].

\section{CONCLUSION}

Recently, machine learning for intrusion detection has received much deliberation. In any IDS, audit data samples are analyzed to set detection rules in highly node - mobile network to protect against number of novel attacks. The primary advantage of using MLbased detection systems is that it is highly accurate and able to detect or categorize attacks without any environmental influence. Different ML-based IDS approaches have their own benefits and disadvantages. Therefore, considering the MANET scenarios, it is important to choose a precise method for implementing IDS. In this paper, one can see that presents the security attacks for the ad hoc network, and machine learning based intrusion detection methods to tackle these attacks and may be proved important for selecting the appropriate methods on bases of the situation in MANET. our future work will focus on proposing a model consists of Deep Q network model. Where Convolutional Neural Networks (CNN) will be trained by the ISCXIDS2012 dataset. After training the model, Deep Q learning algorithm will be used to analyze the network intrusions with the help of network traffic. Our model, will train the CNN with the Intrusion detection dataset.

\section{REFERENCS}

[1] Ehsan Amiria, Hassan Keshavarz, Hossein Heidari, Esmaeil Mohamadi, Hossein Moradzadeh, "Intrusion Detection Systems in MANET: A Review, " ICIMTR International Conference on Innovation, Management and Technology Research, Malaysia, 22 - 23 September, 2013

[2] Safaa Laqtib, Khalid El Yassini, Meriem Houmer, Moulay Driss El Ouadghiri, Moulay Lahcen Hasnaoui,"Impact of mobility models on Optimized Link State Routing Protocol in MANET," Proceedings of the International Conference on Wireless Networks and Mobile Communications (WINCOM), 26-29 Oct. 2016.

[3] B. Awerbuch, D. Holmer, C. Nita-Rotaru, and H. Rubens, "An On-demand Secure Routing Protocol Resilient to Byzantine Failures," Proceedings of the ACM Workshop on Wireless Security, pp. 21-30, 2002.

[4] Bing Wu, Jianmin Chen, Jie Wu, Mihaela Cardei , "A Survey on Attacks and Countermeasures in Mobile Ad Hoc Networks," ,Department of Computer Science and Engineering Florida Atlantic University, WIRELESS/MOBILE NETWORK SECURITY.

[5] Ismail Butun, Salvatore D. Morgera, Ravi Sankar, "A Survey of Intrusion Detection Systems in Wireless Sensor Networks," IEEE Communications Surveys \& Tutorials ,Volume: 16, Issue: 1, 17 May 2013

[6] Salman Iqbal, Miss Laiha Mat Kiah, Babak Dhaghighi, Muzammil Hussain, Suleman khan, Muhammad Khurram Khan, Kim-Kwang Raymond Choo,On Cloud Security Attacks: "A Taxonomy and Intrusion Detection and Prevention as a Service," Journal of Network and Computer Applications Volume 74, October 2016, Pages 98-120 
[7] Raja Waseem Anwar, Majid Bakhtiari, AnazidaZainal, Abdul Hanan Abdullah and Kashif Naseer Qureshi, " Enhanced Trust Aware Routing against Wormhole Attacks in Wireless Sensor Networks," International Conference on Smart Sensors and Application, IEEE.

[8] Saurabh Ughade, R.K. Kapoor, Ankur Pandey, "An overview on Wormhole Attack in Wireless Sensor Network: Challenges, Impacts, and Detection Approach," International Journal of Recent Development in Engineering and Technology, ISSN 2347 - 6435 (Online) Volume 2, Issue4.2014

[9] VarshaGharu, Mahesh Pawar, Jitendra Agarwal , "A Literature Survey on Security Issues of WSN and Different Types of Attacks in Network," Indian Journal of Computer Science and Engineering (IJCSE), 2017

[10] Neellima Singla, Mr.Ramanjeet Singh, "Wormhole Attack Prevention and Detection in MANETs Using HRL Method ”,International Journal of Advance Research, Ideas and Innovations in Technology,2017

[11] Sushama Singh ; Atish Mishra ; Upendra Singh, "Detecting and avoiding of collaborative black hole attack on MANET using trusted AODV routing algorithm," Symposium on Colossal Data Analysis and Networking (CDAN), 18-19 March 2016

[12] Tauseef Jamal ,Shariq Aziz Butt, "Malicious node analysis in MANETS", International Journal of Information Technology, 20 April 2018

[13] Houda Moudni ; Mohamed Er-rouidi ; Hicham Mouncif ; Benachir El Hadadi, "Performance analysis of AODV routing protocol in MANET under the influence of routing attacks, " International Conference on Electrical and Information Technologies (ICEIT), 4-7 May 2016

[14] Desai, V., \& Shekokar,.N, "Performance evaluation of OLSR protocol in MANET under the influence of routing attack. In Wireless Computing and Networking (GCWCN)," IEEE Global Conference on (pp. 138-143). 2014.

[15] Noureldien A. Noureldien, Saeed K. Saeed, M. Ahmed Salih and Alsawi M. Ahmed, "Survey of Mobile Ad hoc Networks Attacks and a New Classification Scheme," British Journal of Mathematics \& Computer Science 8(1): 25-38, 2015,

[16] Ruchita M, Seema L. Review paper on flooding attack in MANET, Int. Journal of Engineering Research and Applications. 2014;4(1).

[17] Rishabh J, Charul D, Meenakshi, “A survey of protocols and attacks in MANET Routing," International Journal of Computer Science and Management Studies. 2012;12(3).

[18] Manjeet Singh, Gaganpreet Kaur.,"A survey of attacks in MANET," International Journal of Advanced Research in Computer Science and Software Engineering. 2013;3(6).

[19] Alomari E, Manickam S, Gupta BB, Karuppayah S, Alfaris R, "Botnet-based distributed denial of service (DDoS) attacks on web servers: classification and art," International Journal of Computer Applications. 2012;49:24-32.

[20] R. Bhuvaneswari, R. Ramachandran ," Denial of service attack mitigation addressing all the security attributes in OLSR MANET," International Journal of Wireless and Mobile Computing ,2018

[21] Ruchi Makani, B.V.R. Reddy, "Taxonomy of Machine Leaning Based Anomaly Detection and its suitability", International Conference on Computational Intelligence and Data Science (ICCIDS 2018).

[22] Robert Mitchell and Ing-Ray Chen., "A Survey of Intrusion Detection in Wireless Network Applications", Elsevier, vol. 42, 1- 23.2014

[23] Ismail Butun, Salvatore D. Morgera, and Ravi Sankar. "A Survey of Intrusion Detection Systems in Wireless Sensor Networks", IEEE Communications Surveys \& Tutorials Volume: 16, Issue: 1,pp266 282.2014

[24] Sumit, S., D. Mitra, and D. Gupta, "Proposed Intrusion Detection on ZRP based MANET by effective k-means clustering method of data mining," 2014. IEEE.

[25] Mohit Soni ; Manish Ahirwa; Shikha Agrawal, "A Survey on Intrusion Detection Techniques in MANET," International Conference on Computational Intelligence and Communication Networks (CICN)12-14 Dec. 2015

[26] Neethu B. "Classification of intrusion detection dataset using machine learning approaches," International Journal of Electronics and Computer Science Engineering; 2012.

[27] Nutan Farah Haq, Musharrat Rafni, Abdur Rahman Onik, Faisal Muhammad Shah, Md. Avishek Khan Hridoy and Dewan Md. Farid, "Application Of Machine Learning Approaches in Intrusion Detection System: A Survey," (IJARAI) Inter- national Journal of Advanced Research in Arti_cial Intelligence, Vol. 4, No.3, pp. 9-18, 2015

[28] Gauthama Raman M R, Nivethitha Somu, Kannan Kirthivasan, Shankar Sriram V S ,"A Hypergraph and Arithmetic Residue-based Probabilistic Neural Network for Classi_cation in Intrusion Detection Systems," Neural Networks, Vol- ume 92, August 2017, pp. 89-97, ELSEVIER.

[29] Aastha Puri and Nidhi Sharma, "A Novel Technique for Intrusion Detection System for Network Security Using Hybrid SVM-CART," International Jour- nal of Engineering Development and Research, Volume 5, Issue 2, ISSN: 2321- 9939, pp. 155-161.2017

[30] Wenjuan Li, Weizhi Meng, Lam-For Kwok, and Horace H. S. IP , "Enhancing Collaborative Intrusion Detection Networks Against Insider Attacks Using Supervised Intrusion Sensitivity-Based Trust Management Model,'Journal of Network and Computer Applications, Volume 77, 1 January 2017, pp. 135-145, ELSEVIER. 
[31] Ch. Nagamani and Suneetha Chittineni , "Network Intrusion Detection Mechanisms Using Outlier Detection, " Second International Conference on Inven- tive Communication and Computational Technologies (ICICCT), pp. 1468-1473,2018.

[32] Eduardo Massato Kakihata, Helton Molina Sapia, Ronaldo Toshiaki Oiakawa, Danillo Roberto Pereira, Joao Paulo Papa, Victor Hugo Costa de Albuquerque and Francisco Assis da Silva , "Intrusion Detection System Based On Flows Using Machine Learning Algorithms, ” IEEE Latin America Transactions, Volume 15, Issue: 10, pp. 1988 - 1993, Oct. 2017.

[33] Parag Verma, Shayan Anwar, Shadab Khan and Sunil B Mane, " Network Intrusion Detection Using Clustering and Gradient Boosting, " 2018 9th Interna- tional Conference on Computing, Communication and Networking Technologies (ICCCNT), Bangalore, 2018, pp. 1-7.

[34] Abdulla Amin Aburomman and Mamun Bin Ibne Reaz, "A novel SVMkNN- PSO ensemble method for intrusion detection system, ” Applied Soft Computing, Volume 38, January 2016, Pages 360-372, ELSEVIER

[35] Jonathon Ng, Deepti Joshi and Shankar M. Banik, “ Applying Data Mining Techniques to Intrusion Detection, " International Conference on Information Technology - New Generations (ITNG), Las Vegas, NV, USA, 2015,pp. 800-801.

[36] Aafreen K. Siddiqui and Tanveer Farooqui , "Improved Ensemble Technique based on Support Vector Machine and Neural Network for Intrusion Detection System, " International Journal Online of Science, Vol. 3, No. 12, pp. 1-8, 2017.

[37] Y. Xin, L. Kong, Z. Liu, Y. Chen, Y. Li, H. Zhu, M. Gao, H. Hou and C. Wang , “ Machine learning and deep learning methods for cybersecurity, ” IEEE Access, pp. 35365-35381, vol. 6, 2018.

[38] Yung-Tsung Hou, Yimeng Chang, Tsuhan Chen, Chi-Sung Laih, Chia-Mei Chen , " Malicious Web Content Detection by Machine Learning, expert system with applications, "Volume 37, Issue 1, January 2010, pp. 55-60, ELSEVIER.Carlos A.

[39] Catania, Facundo Bromberg, Carlos Garc_a Garino (2012), \An Autonomous Labeling Approach to Support Vector Machines Algorithms for Network Tra_c Anomaly Detection," expert systems with applications, Volume 39, Issue 2, 1 February 2012, Pages 1822-1829, ELSEVIER. 